\title{
Instrumental Stability Requirements for Exoplanet Detection with a Nulling Interferometer - Variability Noise is a Central Issue -
}

Bruno Chazelas ${ }^{\left(1,{ }^{*}\right)}$, Frank Brachet ${ }^{\left(1,{ }^{* *}\right)}$, Pascal Bordé ${ }^{\left(2,{ }^{* * *}\right)}$, Bertrand Mennesson ${ }^{(3)}$, Marc Ollivier ${ }^{(1)}$, Olivier Absil ${ }^{(4)}$, Alain Labèque ${ }^{(1)}$, Claude Valette ${ }^{(1)}$, Alain Léger ${ }^{(1)}$

(1) Institut d'Astrophysique Spatiale, CNRS, bat. 121, Univ. Paris-Sud, F-91405 Orsay, France,

(2) Harvard-Smithsonian Center for Astrophysics, 60 Garden Street, MS19, Cambridge, MA 02138, USA

(3) Jet Propulsion Laboratory, California Institute of Technology, 4800 Oak Grove Drive, Pasadena CA 91109-8099 USA

(4) Institut d'Astrophysique et de Géophysique, Université de Liège, Allée du Six Août 17, B4000 Liège, Belgium

$\left({ }^{*}\right) \mathrm{PhD}$ thesis co-funded by Alcatel Space Industry and CNRS

$\left.{ }^{* *}\right) \mathrm{PhD}$ thesis co-funded by Alcatel Space Industry and CNES

$\left({ }^{* * *}\right)$ Michelson Postdoctoral Fellow 
Running title: Variability Noise

Correspondence should be addressed to Alain.Leger@ias.u-psud.fr, tel: 33169858580 , fax: 33169858675 


\section{Abstract}

We revisit the nulling interferometer performances that are needed for direct detection and spectroscopic analysis of exoplanets, e.g. with the DARWIN ${ }^{(1)}$ or TPF-I ${ }^{(2)}$ missions. Two types of requirements are found, one concerning the mean value of the instrumental nulling function, $\langle n l(\lambda)\rangle$, and another one regarding its stability. The former is usually pointed out. It is stringent at short wavelengths but somewhat relaxed at longer wavelengths. The latter, which we call Variability Noise condition, does not usually receive enough attention. It stands whatever the telescope size and the stellar distance are. The results obtained by three nulling experiments, performed in laboratories around the world, are reported and compared with the requirements. All three exhibit $1 / f$ noise that is incompatible with the mission required performances. As already pointed out by Lay ${ }^{(3)}$, this stability problem is not fully solved by modulation techniques. Adequate solutions must be found that likely will include servo systems using the stellar signal itself as a reference, and high stability internal metrology.

Copyright:

OCIS codes: $110.5100 ; 120.3180 ; 350.1260$ 


\section{Nulling Interferometers in the exoplanet research}

The central problem of direct detection of extrasolar planets is the contrast between the star and the planet $\left(410^{7}\right.$ at $7 \mu \mathrm{m}$ in the Sun/Earth case), and the need to cancel the stellar light to analyze the planetary one.

In the IR spectral range, the law of diffraction imposes instruments of several tens of meters, which, in space, can be reached only with diluted pupils. The principle of such an instrument, a nulling interferometer, has been proposed as early as 1978 by Bracewell ${ }^{(4)}$. The idea is to have destructive interferences for the on-axis star, and constructive interferences for an offaxis planet. The fraction of star light that is not cancelled out by the instrument is referred to as the nulling ratio, often characterized by its inverse (the rejection factor).

Rejection factors expected for nulling interferometers (nullers) are of the order of $10^{5}$, significantly less than the star/planet contrast, a few $10^{7}$ at $7 \mu \mathrm{m}$. Consequently, one needs an additional separation of planet and star lights that uses subtraction techniques, and the question of the instrumental stability must be carefully studied. As already stated by Lay ${ }^{(3)}$ in his analytical approach, the null stability drives most of the requirements on the instrument, even if an internal modulation between different sub-interferometers is applied. This statement is confirmed in the present paper and illustrated by means of laboratory experiments.

\section{Noises associated with stellar leakage}

Different processes make the output signal of a nuller non-zero. Even with perfect optics, the instrument's transmission would be zero on-axis only, raising as $\theta^{\alpha}$ off-axis $(\alpha=2,4, \ldots$, according to the interferometer design), where $\theta$ is the angle between the axis and the 
source. A stellar disk having a finite radius, the spatially integrated flux from the star is a fraction, geom, of its total flux (Figure 1). In addition, the instrument is not perfect and transmits a fraction, $n l$, of the on-axis flux. The stellar leakage generate a total photoelectrons flux, at time $t$ and wavelength $\lambda$ :

$$
F_{s l}(\lambda, t)=A \cdot F_{s t}(\lambda) \cdot[g e o m(\lambda, t)+n l(\lambda, t)]
$$

With :

$A$ : a constant depending upon the telescope size, optics throughput, detector yield...; $F_{s t}(\lambda)$ : the incident stellar flux over a spectral bin centered around $\lambda$, in ph-el. $\mathrm{m}^{-2} \cdot \mathrm{s}^{-1}$; geom: the geometrical nulling ratio due to the finite size of the stellar disk and the non-flat response of the interferometer around its axis ( $\theta^{\alpha}$ response), considering perfect optics; geom $<<1$;

$n l:$ the instrumental nulling ratio of the interferometer for an on-axis point source, taking into account instrumental defects; $n l<<1$.

\section{Figure 1}

After an integration time $\tau$, the number of photo-electrons due to stellar leakage is:

$$
\left.N b_{s l}(\tau, \lambda)=A . F_{s t}(\lambda) \cdot[<\operatorname{geom}(\lambda)\rangle_{-} \tau+\langle n l(\lambda)\rangle_{-} \tau\right] \cdot \tau,
$$

where $\langle f\rangle_{-} \tau$ represents the mean value, over the duration $\tau$, of $f$ :

We have

$$
\left.\begin{array}{l}
<\operatorname{geom}(\lambda)>_{-} \tau \equiv \tau^{-1} \int_{0}^{\tau} \operatorname{geom}(\lambda, t) d t \\
<n l(\lambda)>_{-} \tau \equiv \tau^{-1} \int_{0}^{\tau} n l(\lambda, t) d t
\end{array}\right\} .
$$


For a constant stellar flux, both terms in sum (2) introduce noises due to the non-uniform rate of photo-electron generation ("shot noise", also called "quantum noise" or, incorrectly, "photon noise"), and to the stochastic variations of their mean values (their variability for short). We will assume that the pointing of the interferometer towards the star is good enough so that the variability noise of geom would be negligible with respect to its shot noise (However the same analysis developed in this paper could be applied to this variability noise). Because the variability affecting $n l$ finds its origin in the instrument, we call it the instrumental variability noise. It depends on the time variation of $n l(t)$, or $\sigma_{<n \backslash>}(\tau)$, the standard deviation of $\langle n\rangle_{-} \tau$. The total noise associated with $n l$ is therefore the compound effect of shot noise and instrumental variability. In this case, the photo-events form a doubly stochastic Poisson process. Its variance is simply the sum of the shot noise and instrumental variability variances ${ }^{(5)}$.

The shot noise is proportional to the square root of the integrated flux, $\mathrm{Nb}_{\mathrm{sl}}{ }^{1 / 2}$, but the variability noise is directly proportional to $F_{s t}(\lambda)$.

\section{Requirement for the mean rejection factor, $\langle n l(\lambda)\rangle$}

If the interferometer is optimized, at a given wavelength, for a planet position with a relative transmission of unity (planet on a bright fringe), after an integration $\tau$, the signal due to the planet is $A F_{p l} \tau$. As the photon flux due to the planet is much weaker than that due to the stellar leakage, the shot noise is given by the square root of the number of photo-electrons, $\mathrm{Nb}_{\mathrm{sl}}$. The Signal to Noise Ratio (SNR), if the shot noise were the only source of noise, would be

$$
S / N_{s h}=(A \cdot \tau)^{1 / 2} \cdot F_{p l} /\left[\left(F_{s t} \cdot(<g e o m>+<n l>)\right]^{1 / 2},\right.
$$

which improves with integration time as $\tau^{1 / 2}$. 
The shot noise associated with $\langle n l>$ is instrument dependent, whereas that associated with $<$ geom $>$ is intrinsic to an interferometer design and to the stellar angular size, and therefore cannot be avoided. The maximum requirement for $\langle n l>$ results: it should be somewhat smaller than $\langle$ geom $>$, so that the total shot noise would not be significantly increased by the contribution of $\langle n>$. The actual requirement is dictated by the total SNR needed at the corresponding wavelength and depends upon the planetary signal intensity and the other sources of noises, e.g. the Zodiacal light, the thermal background...

At a given wavelength, $\lambda$, the first transmission maximum of a Bracewell interferometer, with base $L$, is at an angle $\theta=\lambda /(2 L)$. This base can be selected so that the target planet is located at one of the transmission maxima. The angular separation of a Sun-Earth system at 20 parsecs (65 light-years) is $\theta=0.05$ arcsec. When observing at $7 \mu \mathrm{m}$, the planet will be located on the first transmission maximum if the interferometer's baseline is $L=14 \mathrm{~m}$. The geometrical stellar leakage due to the finite size of the stellar disk is then geom $=1.810^{-5}$. The most interesting target planets are located in the Habitable Zone ${ }^{(6)}(\mathrm{HZ})$ of their stars, i.e. in the region where water can be liquid (roughly 0.7-1.5 AU for the Sun, i.e. zone between Venus and Mars). Most stars in the DARWIN/TPF target lists are cooler than the Sun ${ }^{(7)}$ and have a larger ratio between their angular size and the distance to their $\mathrm{HZ}$. They produce larger leaks when a planet is searched for in their HZs. Therefore, the preceding value for geom can be considered as the lowest one, and the condition derived thereafter for $\langle n|>$ as the most severe one.

At short wavelengths, i.e. around $7 \mu \mathrm{m}$, the planetary signal is weak, whereas this is an important domain to detect $\mathrm{H}_{2} \mathrm{O}$ and $\mathrm{CH}_{4}$ (Figure 2). Consequently, it is critical that the instrument does not induce a significant additional noise contribution. A possible requirement would be $\langle n|>=0.56\langle$ geom $>$, which corresponds to an increase of the total shot noise by 25 $\%$. For an interferometer with leakages similar to those of a Bracewell interferomter (geom $=1.810^{-5}$ for an Earth-Sun like system), a sensible value for the mean instrumental leakage would then be

$$
<n l>(7 \mu m)=1.010^{-5}
$$


For another interferometer design with more intrinsic leakages, the requirement on $<n />$ could be relaxed, but the required integration time, for a given telescope collecting area, would increase due to the shot noise associated to the larger value of geom.

\section{Figure 2}

At long wavelengths, the requirement on $\langle n|>$ can be relaxed because:

i. the $F_{\text {star }} / F_{p l}$ ratio diminishes, with an analytical upper estimate, in the $7-20 \mu m$ domain of $\left[F_{\text {star }} / F_{p l}\right](\lambda)<\left[F_{\text {star }} / F_{p l}\right](7 \mu \mathrm{m}) \cdot[\lambda / 7 \mu \mathrm{m}]^{-3.37}$ (Figure 3);

ii. other sources of shot noise become important ${ }^{(1)}$, e.g. the flux of the Local (Solar) Zodiacal light, the thermal emission of optics...

\section{Figure 3}

The SNR due to the shot noise should have the same value as at $7 \mu \mathrm{m}$. Considered alone, point (i), leads to the condition:

$$
<n />/ \operatorname{geom}(\lambda)=\left[1.56\left(F_{p l}(\lambda) / F_{p l}(7 \mu m)\right)^{2} /\left(F_{s t}(\lambda) / F_{s t}(7 \mu m)\right)\right]-1,
$$

which reduces to relation (5) when $\lambda=7 \mu \mathrm{m}$ and geom $=1.810^{-5}$.

To prepare the discussion of variability noise, it is convenient to use a more stringent condition, $<n />/$ geom $(\lambda)=0.56\left[\left(F_{p} / F_{s t}\right)(\lambda)\right] /\left[\left(F_{p} / F_{s t}\right)(7 \mu m)\right]$, (Figure 4). This condition should not be very difficult to achieve if that at $7 \mu \mathrm{m}$ is fulfilled. In the case of an optimized Bracewell interferometer ( $g e o m=1.810^{-5}$ ), it can be estimated as

$$
<n l>(\lambda)=1.010^{-5}(\lambda / 7 \mu m)^{3.37} .
$$




\section{Figure 4}

\section{Remarks:}

- Most of the stars, in the DARWIN/TPF-I target lists, are less luminous than the Sun and correspond to a lower $\mathrm{F}_{\mathrm{star}} / \mathrm{F}_{\mathrm{pl}}$ ratio, for an Earth-size planet at $\mathrm{T} \sim 300 \mathrm{~K}$. Consequently, the Sun/Earth case is the most demanding for the nulling instrument.

- We think that it is not possible to optimize the DARWIN/TPF-I instrument at $6 \mu \mathrm{m}$ for a $300 \mathrm{~K}$ planet, because the $F_{p} / F_{s t}$ for such an object is then extremely low (Figure 3 ). However, the performances of the nuller, at $6 \mu \mathrm{m}$ should be kept as good as at $7 \mu \mathrm{m}$ $(<n l>(6-7 \mu \mathrm{m})=\langle\mathrm{nl}>(7 \mu \mathrm{m}))$ in order to be able to study somewhat warmer planets, e.g. planets at $350 \mathrm{~K}$.

- The above conclusion concerns exclusively shot noise, and holds even if the average instrumental stellar leakage is removed by means of a modulation technique. This contribution acts as a bias that cannot be calibrated analytically and hamper the detection of the planet. If no subtraction/modulation technique were used, the null depth needed for a detection should be significantly deeper than the planet/star contrast of $1 / 410^{7}=2.510^{-8}$ at $7 \mu \mathrm{m}$, a very difficult goal indeed. In practice, modulation techniques such as rotation of the $\operatorname{array}^{(4)}{ }^{(8)}$ or phase chopping techniques ${ }^{(9)(10)(11)(12)}$ should remove this bias and allow a detection even if the mean null is not so deep. However, these techniques are affected by null depth fluctuations at all frequencies as proven by Lay ${ }^{(3)}$ : systematic errors are not completely removed by modulation, and the actual requirements on the null will indeed be driven by the requirements on the stability, i.e. variability noise, as discussed hereafter. 


\section{Requirements for Variability Noise}

\subsection{Signal to noise ratio due to Variability Noise}

Assuming that the relative transmission of the instrument for the planetary signal is unity, after integration during time $\tau$, the signal is:

$$
S=A \cdot F_{p l}(\lambda) \cdot \tau
$$

Let us remind the reader that variability noise, $N_{v}$, is due to the stochastic variation of the mean flux at the output of the (imperfect) nuller. It is proportional to the stellar flux and to the standard deviation of the mean of the instrumental nulling ratio $\langle n l>$ over the integration time $\tau, \sigma_{<n \mid>\_}$noted thereafter $\sigma_{<n \mid>}(\tau)$, (see equation (3)).

$$
N_{v}=A \cdot F_{s t}(\lambda) \cdot \sigma_{<n l>}(\tau) \cdot \tau \text {. }
$$

The resulting SNR, is:

$$
S / N_{v}=\left[F_{p l} / F_{s t}(\lambda)\right] \cdot\left[1 / \sigma_{<n l>}(\tau)\right]
$$

The incidence of the integration time, $\tau$, on the SNR through $\sigma_{<n />}(\tau)$ is a key element. Conversely, if a minimum SNR is needed to obtain useful scientific information, a requirement for $\sigma_{<n />}(\tau)$, results.

We can relate $\sigma_{<n l>}(\tau)$ and the PSD of $n l$. We assume that $n l$ is an ergodic random process (ergodic random function of time), which means that its average and moments taken over the time are the same as when taken over the different realizations. The output of an 
instrumental nuller is a function of time having a finite duration $T$. From this output, we estimate the statistical properties of the random process.

This random process has no finite energy in the sense of signal processing, $\int_{0}^{\infty} n l^{2}(t) d t \rightarrow \infty$, but has a finite power, $\lim _{T \rightarrow \infty} T^{-1} \int_{0}^{T} n l^{2}(t) d t$, and a Power Spectral Density (PSD), noted $P S D_{n 1}$

$$
P S D_{n l}(v) \approx \lim _{T \rightarrow \infty} T^{-1}\left|\int_{0}^{T} n l(t) e^{-2 \pi i v t} d t\right|^{2}
$$

Such a PSD, when defined as a limit, follows the standard Fourier transform properties (for details see Léna ${ }^{(13)}$ and Goodman ${ }^{(5)}$ )

To simplify calculation we introduce the running average over $\tau$, a random process noted $<n l>\_(t)$ :

$$
<n l{ }_{-} \tau(t)=\tau^{-1} n l(t) * \Pi(t / \tau),
$$

where $\Pi(u)$ is the top-hat function $(\Pi(u)=1$ for $u$ between 0 and 1 and 0 elsewhere), and $*$ represents the convolution. We have :

$$
<n />_{-} \tau(0)=<n />_{-} \tau
$$

As $\langle n \mid\rangle_{-} \tau(t)$ is a linearly filtered ergodic process it is also ergodic ${ }^{(5)}$. Thus $\sigma_{<n l\rangle}(\tau)$, the standard deviation of $\langle n \mid\rangle_{-} \tau$ for different outcomes of the random process, is equal to the standard deviation of the random process $\langle n \mid\rangle_{-} \tau(t)$ over the time.

Using the Parseval theorem one shows ${ }^{(5)}$ that 


$$
\sigma_{<n l\rangle}^{2}(\tau)=\int_{-\infty}^{\infty} P S D_{\langle n l\rangle_{-} \tau}(v)-\langle n l\rangle^{2}
$$

As $\langle n l\rangle_{\_} \tau$ has a non zero mean value, its PSD has a singular behavior in $v=0$. It goes as $\left\langle n />^{2} \delta(v)\right.$, where $\delta(v)$ is the delta "function". This is because the Fourier transform at $v=0$ is the mean value of the function. The PSD' function, which is equal to PSD but in zero, defined as:

$$
P S D_{\langle\tau\rangle_{-} \tau}^{\prime}(v)=P S D_{\langle\tau\rangle_{-} \tau}^{\prime}(v)-\langle n l\rangle^{2} \delta(v)
$$

has a non-singular behavior in 0 , and

$$
\sigma_{<n l>}^{2}(\tau)=\int_{-\infty}^{\infty} P S D_{\langle n t\rangle_{\tau} \tau}^{\prime}(v)
$$

The Fourier transform of $\Pi(t)$ being $\operatorname{sinc}(v) \equiv \sin (\pi v) / \pi v$, then $\sigma_{<n />}(\tau)$ derives from the Power Spectral Density of the nulling function $P S D_{n l}$ :

$$
\sigma_{<n l>}^{2}(\tau)=\int_{-\infty}^{\infty} P S D_{n l}^{\prime}(v) \operatorname{sinc}^{2}(\tau v) \mathrm{d} v
$$

If $\mathrm{PSD}_{\mathrm{nl}}{ }_{\mathrm{l}}$ is decreasing rapidly on the scale $\Delta v=\tau^{-1}$, most of the contribution to the integral is around zero. Then, $\operatorname{sinc}(v)$ can be approximated by a top-hat function with $\mathrm{FWHM}=\tau$, and equation (11) reads

$$
\sigma_{<n l>}^{2}(\tau) \approx \int_{-0.5 / \tau}^{0.5 / \tau} P S D^{\prime}{ }_{n l}(v) \mathrm{d} v
$$

If the fluctuations of $n l$ are white, i.e., $P S D_{n l}(v)$ is constant, the familiar result is found, $\sigma_{<n l}(\tau)$ is proportional to $\tau^{-1 / 2}$ and $S / N_{v}$ increases as $\tau^{1 / 2}$.

If there are instrumental drifts, the PSD of the nulling ratio will have a so-called " $1 / f$ " component. This does not mean that $P S D_{n l}$ is diverging in 0 . It means that the longer the time 
interval between two calibrations of the instrument, the more noise power at low frequency. Let $T$ be the time separating two calibrations of the nuller. A possible model of this noise is :

$$
P S D_{n l}(v)=\left\{\begin{array}{ccc}
a v^{-1}+b & \text { for } & |v|>T^{-1} \\
a T+b & \text { for } & |v| \leq T^{-1}
\end{array}\right.
$$

This model (Figure 5) gives a better representation of the physical reality, with a finite energy for any finite value of $T$. Integrals in equation (12) or (11) are defined.

What is the effect of increasing the integration time $\tau$, on the $S / N_{v}$ ? In order to compare different measurements with integration times $\tau$, e.g. some with an interferometer orientation yielding the planetary signal and other ones not doing so, the time $T$ between two calibrations, must be several times $\tau$. Applying equation (12), one gets :

$$
\begin{gathered}
\sigma_{<n l>}^{2}(\tau) \approx 2 \int_{0}^{0.5 \tau^{-1}} P S D^{\prime}{ }_{n l}(v ; T) d v \approx 2 \int_{0}^{T^{-1}}(a T+b) d v+2 \int_{T^{-1}}^{0.5 \tau^{-1}}\left(a v^{-1}+b\right) d v \\
\sigma_{<n l>}^{2}(\tau) \approx 2 a\left(1+\ln \left(\frac{T}{2 \tau}\right)\right)+\frac{b}{\tau}
\end{gathered}
$$

If $a \tau \gg b$, the " $1 / f$ " noise is dominating the integral, and :

$$
\sigma_{<n l>}^{2}(\tau) \approx 2 a\left(1+\ln \left(\frac{T}{2 \tau}\right)\right)
$$

which is very slowly decreasing with $\tau$. The reduction of the integration domain is mostly compensated by an increase of the PSD in the low frequency part. In presence of a dominating $1 / f$ noise, the $S / N$ is almost independent of the integration time $\tau$.

If $a \tau<<b$, white noise is dominating, and $\sigma_{<n l>}^{2}(\tau) \approx b / \tau$. The familiar result is met, $\sigma_{<n \mid>}(\tau)$ is proportional to $\tau^{-1 / 2}$ and $S / N_{v}$ increases as $\tau^{1 / 2}$. 


\subsection{Nulling experiments around the world}

Considering a few nulling experiments presently performed around the world, is informative. For several of them, the authors kindly provided us with the data files of their experimental $n l(t)$ function. We have computed the running average $\left\langle n l{ }_{-} \tau(t)\right.$ over $\tau$, the PSD of $n l$ and the standard deviation for several integration times $\tau$.

The results are shown in Figure 6, 7 and 8. For the best results (Figs.7 \& 8) the mean values miss the goal of $10^{-5}$ by a factor of 5 and 2 , respectively. For $\tau=10 \mathrm{~s}$, they yield $\sigma_{<n l>}(10 \mathrm{~s}) \sim 10^{-6}$. At $7 \mu \mathrm{m}$, the Sun-to-Earth flux ratio, $F_{p l} / F_{s t}$ is $2.510^{-8}$. If this were the final result, the $S / N_{v}$ value resulting from (10) would be $\sim 2.510^{-2}$, whatever the stellar distance and the telescope diameters, clearly an unacceptable situation.

\section{Figure 6 ,Figure $7, \underline{\text { Figure } 8}$}

Fortunately, longer integrations are expected to improve this SNR. If variability noise were white, i.e. had a constant PSD, relation (12) predicts that, with increasing time, $\sigma_{<n>>}(\tau)$ would decrease as $\tau^{-1 / 2}$. Starting from the nuller performances reported for $\tau=10 \mathrm{~s}, S / N_{v}$ would increase to $2.510^{-2}(24 \times 3600)^{1 / 2} \sim 7$ at $7 \mu \mathrm{m}$, after a 10-day integration.

Unfortunately, all present experimental PSDs exhibit a 1/f-type peak at low frequencies. Consequently, the standard deviations of $n l$ decrease slower than $\tau^{-1 / 2}$ and extrapolation to very long integration durations (days) does not look promising. These $1 /$ f noises are probably due to drifts in the experimental setups.

The issue of long-term drift is of major importance. Although this drift is difficult to control, the situation is not hopeless. For instance, an important limitation for the instrumental nulling 
ratio stems from the fine control of the Optical Path Difference (OPD) between the different arms of the interferometer. Now, the servo adjustment of the delay line around a zero OPD on DARWIN/TPF-I instruments will be performed using the stellar signal itself (fringe tracker in the visible/near-IR) and should have no long-term drifts. However, an adequate stability will be required for the instrument parts downstream the fringe tracker. The other limiting factors of $n /$ should be controlled as well, including intensity errors, pointing errors...

\subsection{Required performances}

When the noises associated with the stellar leakage (relation (1)) are dominant, e.g. at short wavelengths, a possible noise budget is

$$
N_{v}=N_{s h}=(1 / 2)^{0.5} \cdot N_{t o t} .
$$

To obtain spectroscopic information, at different wavelengths, including the most difficult ones, the $S / N_{\text {tot }}$ ratio must be sufficient in a maximum of 10 day integration, say $S / N_{\text {tot }} \sim 7$. Relation (13) implies

$$
S / N_{v}=(1 / 2)^{-0.5} S / N_{t o t} \sim 10 \text {, in } 10 \text { days. }
$$

At $7 \mu \mathrm{m}$, the star to planet contrast is $410^{7}$ for the Earth/Sun case. At other wavelengths, the relation $F_{s t} / F_{p l}(\lambda)$ can be deduced from Fig.3, or estimated from the $(\lambda / \mu m)^{-3.37}$ relation. Equation (10) implies a requirement for the nuller stability that is shown in Fig.9. In the 7$20 \mu \mathrm{m}$ domain, it can be written as:

$$
\sigma_{<n \mid>}(\lambda, 10 \text { days }) \leq 2.510^{-9}(\lambda / 7 \mu m)^{3.37}
$$

or

$$
\sigma_{<n 1>}(\lambda, 10 s) \leq 710^{-7}(\lambda / 7 \mu m)^{3.37}+\text { white noise. }
$$

\section{Figure 9}


The condition "white noise" means that $1 / f$ type noises, e.g. long term drifts, must be small enough to permit an improvement of stability with integration time as $\tau^{-1 / 2}$.

If the performance on the mean value is achieved $\left(\langle n /\rangle=10^{-5}\right.$ at $\left.7 \mu \mathrm{m}\right)$, that on its stability can be expressed in terms of relative values:

$$
\left[\sigma_{<n \mid>} /<n \mid>\right](10 \text { days }) \leq 2.510^{-4}
$$

or

$$
\left[\sigma_{<n \mid>}|<n|>\right](10 s) \leq 710^{-2}+\text { white noise }
$$

The long-term (10-day) instrumental stability is very demanding, especially at short wavelengths. These requirements are independent of the target distance and the telescope collecting area.

The laboratory demonstration of a nuller with these performances can be divided into two steps:

1. Obtaining the requirements for short integration times, e.g. $10 \mathrm{~s}$;

2. Showing that the question of the long-term stability, i.e. the absence of drifts (1/f type noises), can be dealt with. This point is shortly discussed in the next section.

\section{How to beat the 1/f component of the Variability Noise?}

In this paper, for the sake of simplicity, we have discussed only the case of a single interferometer. To beat the drift problem, modulation techniques such as rotation of the array $^{(4)(8)}$ or phase chopping techniques ${ }^{(9)(10)(11)(12)}$ have been proposed. As shown by Lay ${ }^{(3)}$, 
they improve the situation but do not fix it. For instance, Lay showed that cross terms between phase errors and amplitude errors are not eliminated by those techniques.

Another way to understand this limitation is to note that in most cases - all of them, up to now - the different sub-interferometers have independent instrumentations, e.g. phase shifters. Then they have independent drifts and switching from one to another does not eliminate these drifts. A solution could be to use the same optics downstream from the fringe tracker for the different sub-interferometers, if such a design exists.

As a general remark, 1/f-type noises (drifts) are beaten down by servo-loops (end of Sect.4.2), so these should be used wherever possible.

\section{Conclusions}

We have revisited the performances required for a central part of the optical instrument needed for DARWIN or TPF-I missions, the nulling interferometer. Two types of requirements are found, one for the mean value of its nulling ratio (or rejection factor), $n l(\lambda, t)$, and another for its stability. The former is usually pointed out, but, with the exception of Lay ${ }^{(3)}$, insufficient attention is paid to the latter that we call "variability noise", probably the most demanding one. In this paper we have neglected the variability of the instrument pointing. This noise contribution is possibly as severe as the nulling variability noise and should be carefully studied.

Regarding the mean value of the stellar leakage, for a Bracewell-type interferometer, a possible condition is shown in Fig.4. A more severe condition is given by equation (7) and the following remarks. At short wavelengths, this condition is demanding and needed because this is an important spectral domain (short-wavelength signature of $\mathrm{H}_{2} \mathrm{O}$, and that of $\mathrm{CH}_{4}$ ). At longer wavelengths, the condition is easier to meet. 
In the $7-20 \mu \mathrm{m}$ domain, the condition on the null stability can be written as in equation (15) and (17). It must be noted that these requirements stand whatever the telescope size and the stellar distance are. This stability of the mean nulling ratio is required in order to separate the stellar leakage from the planetary signal when a subtraction technique is used.

Three nulling experiments, performed in laboratories around the world are reported. The best two miss the requirement on the mean nulling by a factor of 2 to 5 , and approach, or reach, the short-term (10s) stability requirement. Most important, all of them show clear evidence of drifts on longer integration times that prevent a sufficient increase of the SNR with integration time. However, it must be noted that the experiments reported here are only first steps in a process aiming at the validation of nulling interferometry techniques for DARWIN/TPF missions. In particular, they were not built to have the highest stabilities, but to have as deep as possible nulls, and some were pretty successful within that respect. They were operated at $\mathrm{CO}_{2}$ laser wavelength or shorter wavelengths $(\sim 1.5 \mu \mathrm{m}$, i.e. the telecom domain), for convenience. This is a first step, but experiments at relevant wavelengths $(6-20 \mu \mathrm{m})$ are needed, with improved performances.

A central problem is the long-term stability of instrument outputs. In particular, as shown by Lay $^{(3)}$, the presently proposed internal modulations between sub-interferometers with different base orientations ${ }^{(8)(9)(10)(11)}$, do not fully solve this question of stability because they use independent nulling optical devices, with the corresponding differential drifts. We think that special attention should be paid to servo systems that use the stellar signal itself, at shorter wavelengths, as a reference to monitor key quantities as the OPD, because these systems should be free of long term drifts.

\section{Acknowledgments:}


The authors are grateful to Luigi d'Arcio, Anders Karlsson, Oliver Lay, Nick Woolf and Yves Rabbia for valuable exchanges; and to Alcatel Space Industry and Astrium Germany for kindly providing the data files of their nulling experiments. BC and FB are grateful to Alcatel Space Industry, and CNRS and CNES, respectively, for funding their thesis grant. This work was performed in part under contract 1256791 with the Jet Propulsion Laboratory (JPL) funded by NASA through the Michelson Fellowship Program. JPL is managed for NASA by the California Institute of Technology. OA acknowledge the support of the Belgian FNRS.

\section{References}

1. M., Fridlund, 2000, "DARWIN, Concept and Feasibility Study Report", ESA-SCI (2000) 12, chapter 3, p 1-218

2. P.R., Lawson and J. A., Dooley, 2005, "Technology Plan for the Terrestrial Planet Finder Interferometer", JPL Publication 05-5, p 1-163

3. O.P., Lay, 2004, "Systematic errors in nulling interferometers", Applied Optics, 43, p 61006123

4. R.N., Bracewell, 1978, "Detection of non solar planets by spinning infrared interferometer", Nature, 274, p 780-781

5. J. W., Goodman, 2000, "Statistical Optics", Wiley Classics Library, 2000 p. 466-468, p 
6. J. F., Kasting, D. P., Whitmire, R. T., Reynolds, 1993, Habitable Zones around Main Sequence Stars, Icarus, Volume 101, Issue 1, p 108-128

7. A., Stankov, L., Kaltenegger, C., Eiroa, 2005,ESA/ESTEC internal publication DMS/SCIA/DARWIN/208.

8. B., Mennesson and J-M., Mariotti, 1997, "Array configurations for a space infrared nulling interferometer dedicated to the search for Earthlike extrasolar planets", Icarus, Volume 128, p 202-212

9. J.R.P., Angel and N.J., Woolf, 1997, "An Imaging Nulling Interferometer to Study Extrasolar Planets", Astrophys. J, 475, p 373-379

10. A., Karlsson and B., Mennesson, 2000, "The Robin Laurance nulling interferometers", Proceedings SPIE 4006, p 871-874

11. O., Absil , A., Karlsson and L., Kaltenegger, 2003, "Inherent modulation: a fast chopping method for nulling interferometry", Proceedings SPIE 4852, p 431-435.

12. B., Mennesson, A., Léger and M., Ollivier, 2005, " Direct Detection and Characterization of Extrasolar Planets: the Mariotti Space Interferometer", Icarus (in press)

13. P., Léna, F., Lebrun and F., Mignard, 1998, Observational Astrophysics, Springer, p 453

14. M., Ollivier, 1999, "Contribution à la recherche d'exo-planètes. Coronographie interférentielle pour la mission DARWIN", PhD, Université de Paris-Sud, Orsay

15. M., Ollivier, J-M., Mariotti, A., Léger, P., Sekulic, J., Brunaud, G., Michel, 2001, "Interferometric Coronography for the DARWIN Space Mission - Laboratory Demonstration Experiment -", A\&A 370, p 1128-1136 
16. R., Flatscher, Z., Sodnik, K., Ergenzinger, U., Johann and R., Vink, 2003, "DARWIN nulling interferometer breadboard I: system engineering and measurement", eds. M., Fridlund and T., Henning, ESA publication SP-539, p 283-291 


\section{Figure captions}

Figure 1: transmission of a nulling interferometer with a $\theta^{2}$ transmission around its axis. The finite size of the stellar disk leads to leaks with a relative intensity, geom.

Figure 2: spectrum of a planet with $\mathrm{H}_{2} \mathrm{O}$ and $\mathrm{CH}_{4}$ in their atmospheres ${ }^{(1)}$. The 7-9 $\mu \mathrm{m}$ range is important to detect these two important species.

Figure 3: ratio of the Sun to Earth fluxes, vs. wavelength, normalized at $7 \mu \mathrm{m}$. The fluxes are approximated by Planck functions at $5800 \mathrm{~K}$ and $288 \mathrm{~K}$, respectively. In the $7-20 \mu \mathrm{m}$ domain, the power law curve $(\lambda / 7 \mu \mathrm{m})^{-3.37}$ can be used as an upper value for this ratio.

Figure 4: condition on $\langle n l(\lambda)\rangle$, imposed by the shot noise, for a Bracewell interferometer $\left(\right.$ geom $\left.=1.810^{-5}\right)$. The condition is the most severe at $7 \mu \mathrm{m}$, where we required that the noise due to the instrumental stellar leakage does not increase significantly the noise due to the intrinsic stellar leakage (geom). At longer wavelengths, the actual requirement is that of the $\langle n l(\lambda)>$ curve (relation (6)). For preparing the discussion on the standard deviation of the variability noise (section 4), it is convenient to impose the more stringent condition $<n l(7 \mu m)]>x\left[\left(F_{p} / F_{s t}\right)(\lambda)\right] /\left[\left(F_{p} / F_{s t}\right)(7 \mu m)\right]$, or its analytical estimate $\left.<n l(7 \mu m)\right]>x[\lambda / 7 \mu m]^{3.37}$

Figure 5: llustration of the mathematical model used to describe the "1/f" noise. It has a finite power, but this power grows with the time $T$, the time separating 2 calibrations of the instrument.

Figure 6: results from the nulling experiment by Ollivier and co-workers ${ }^{(14),(15)}$ in 1999, using a $\mathrm{CO}_{2}$ laser $(10.6 \mu \mathrm{m})$. From top to bottom, (1) experimental nulling function, $\mathrm{nl}(t)$, versus time ; (2) running average over 10s duration; (3) Power Spectral Density (PSD) of the nulling function $n l(t)$.The increase of the PSD for low values of $v$ is clear. In the range $(0.3-0.01$ 
$\mathrm{Hz}$ ), the PSD goes approximately as $v^{-1.35}$, a behavior close to the canonical $1 / \mathrm{f} \equiv 1 / v$ one; (4) standard deviation of the mean value of $n l$ over time $\tau, \sigma_{<n>}(\tau)$. In the frequency range investigated by that experiment, $\sigma$ decreases with $\tau$, but more slowly than $\tau^{-1 / 2}$, which typical of PSD's with 1/f like components.

Figure 7: results from the nulling experiment by Alcatel (2004), using a laser diode at $\sim 1.57 \mu \mathrm{m}$ and an integrated optics recombiner. From top to bottom, (1) experimental nulling function, versus time, $\mathrm{nl}(\mathrm{t})$; (2) running average over $3 \mathrm{~s}$ duration; (3) The corresponding PSD in the $(0,8 \mathrm{~Hz})$ range. Its increase at low frequencies is clear. From 0.1 to $1 \mathrm{~Hz}$ it goes approximately as $1 / v ;(4)$ standard deviation of the mean value of $n l$ over time $\tau, \sigma_{<n l>}(\tau)$. The behavior of $\sigma$ with the integration time is similar to that in Fig.6 and indicates that major improvements in experimental set-up are needed to obtain the required stability i.e. a $\tau^{-1 / 2}$ decrease with integration time. (Courtesy Alcatel Space Industry, 2004)

Figure 8: quantities similar to those in Fig.6 and 7, from the Astrium experiment in $2004{ }^{(16)}$. The duration of the experiment is the longest of the three. The null curve between $1000 \mathrm{~s}$ and $1500 \mathrm{~s}$ is selected to compute the PSD and $\langle n>$ standard deviation, because it is the best quality part. A low frequency increase of the PSD is present. From 0.01 to $0.1 \mathrm{~Hz}$ it goes approximately as $1 / v$. It must be emphasized that this experimental set-up, as well as the two preceding ones, had the goal of a low null value but not that of its stability. Their authors have precise ideas on how to improve this stability. (Courtesy Astrium, Germany)

Figure 9: required stability of the instrumental nulling function, $n l(t)$, on timescales $\tau=10 \mathrm{~s}$ and 10 days. The levels achieved, at shorter wavelengths, in experiments reported in Fig.7 and 8 are indicated. 
Transmission
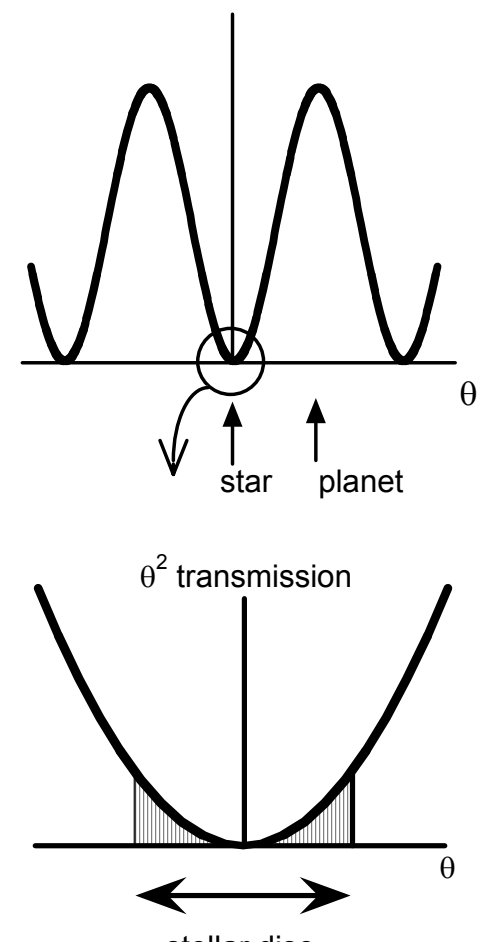

stellar disc

Figure 1 


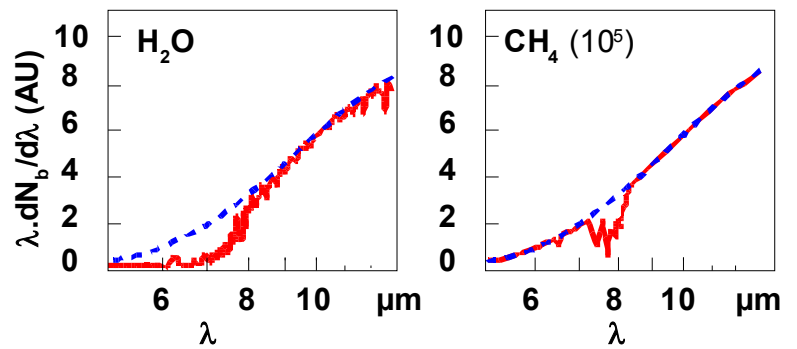

Figure 2 


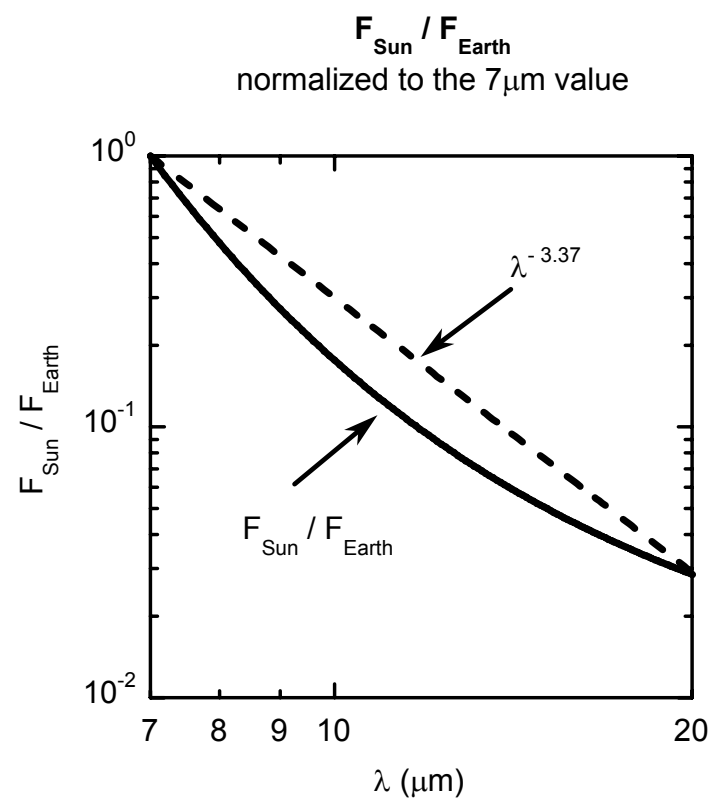

Figure 3 


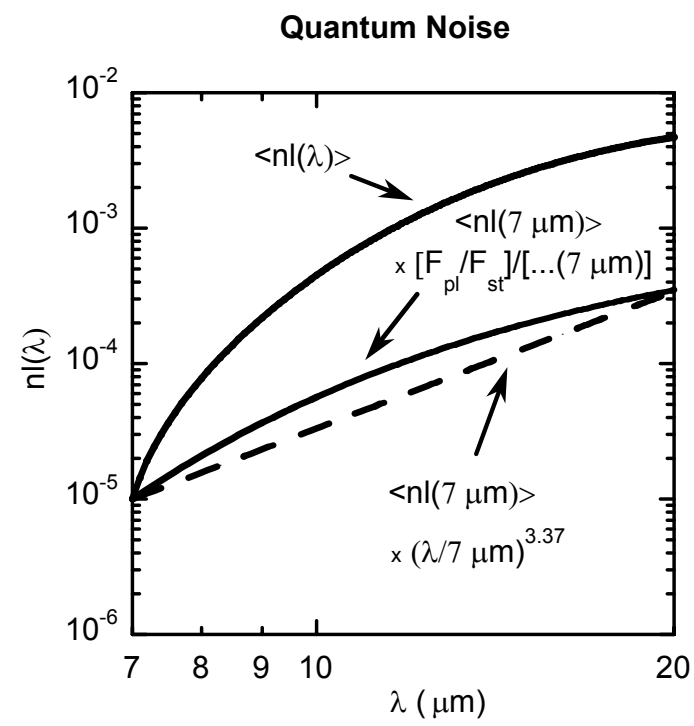

Figure 4

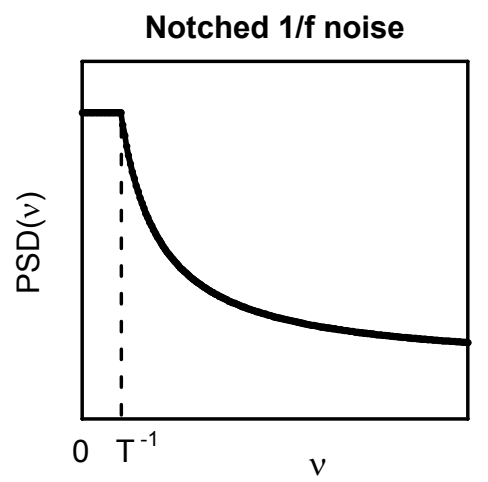

Figure 5 

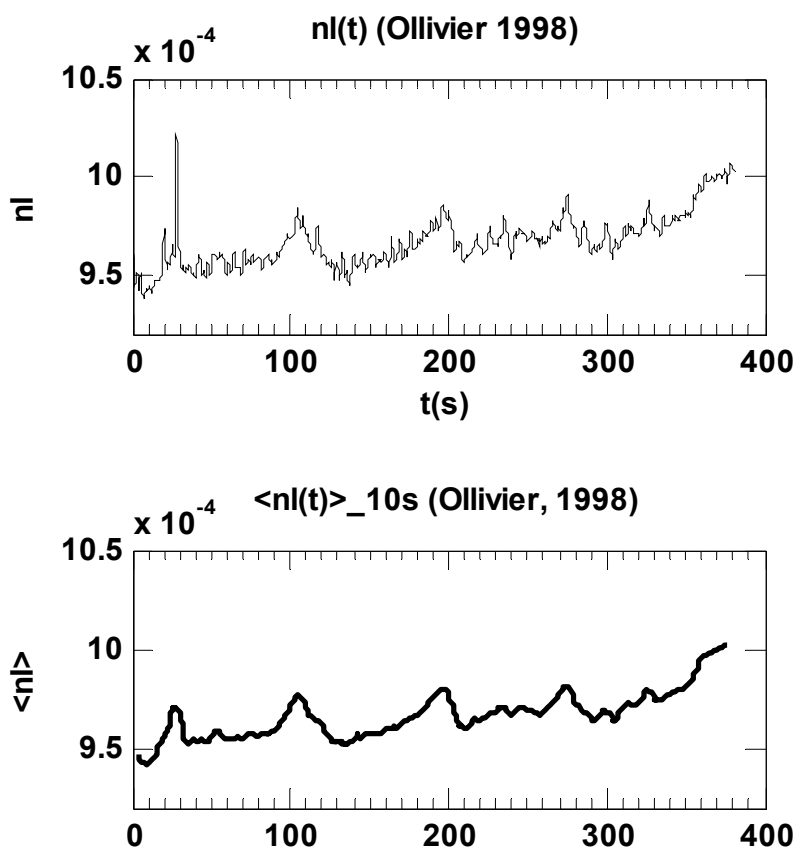

t(s)
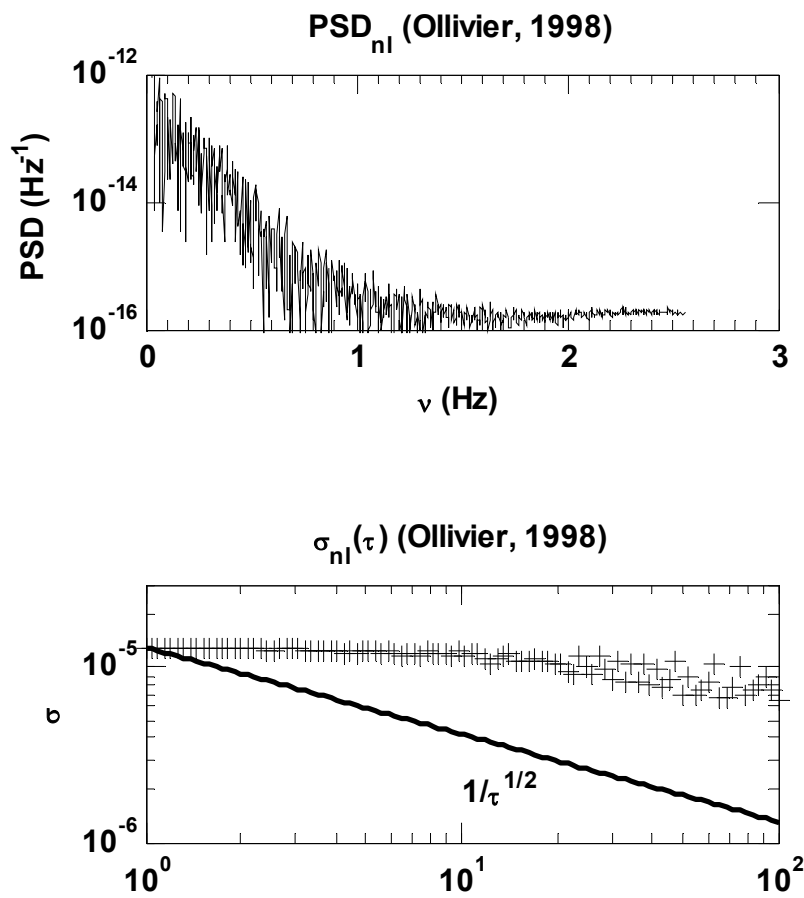

$\tau$ (s)

Figure 6 

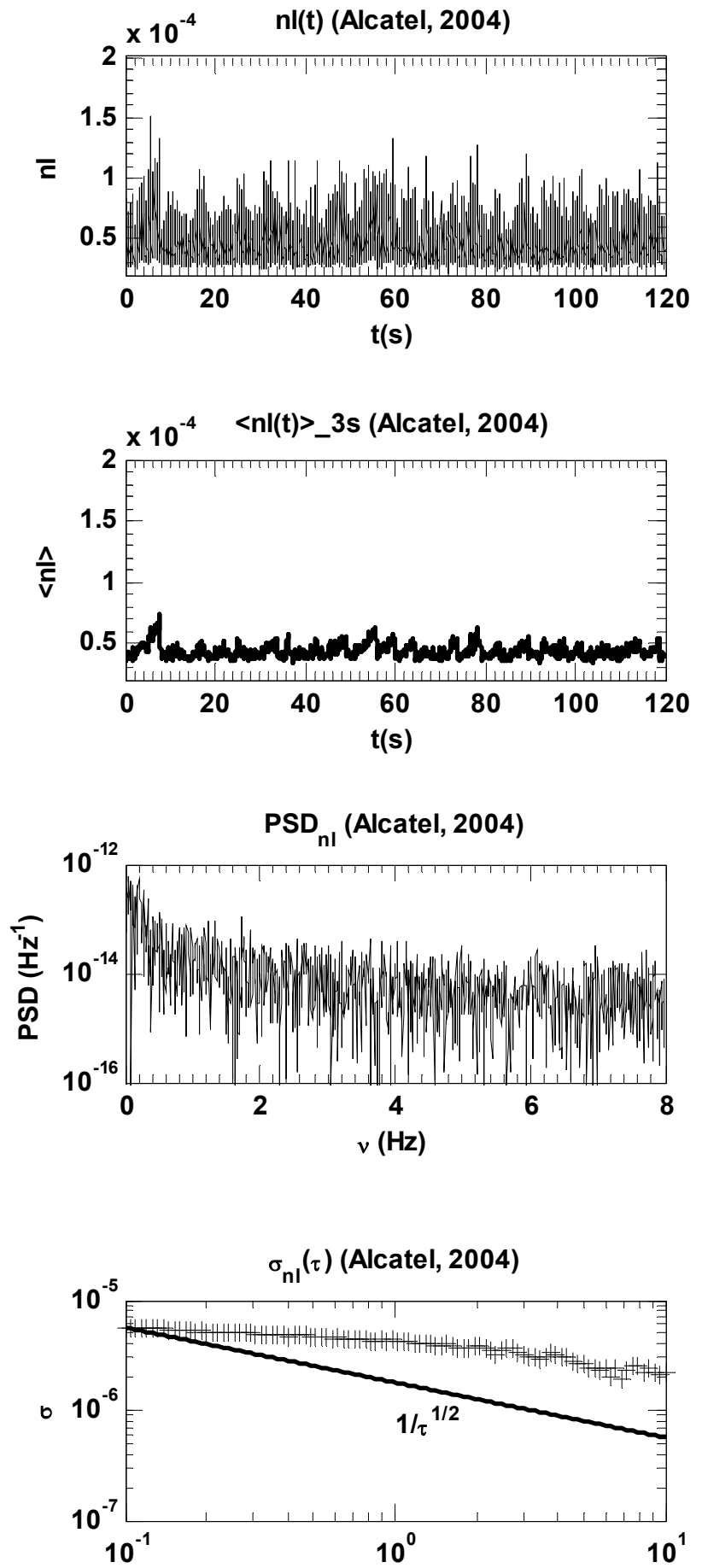

$\tau$ (s)

Figure 7 

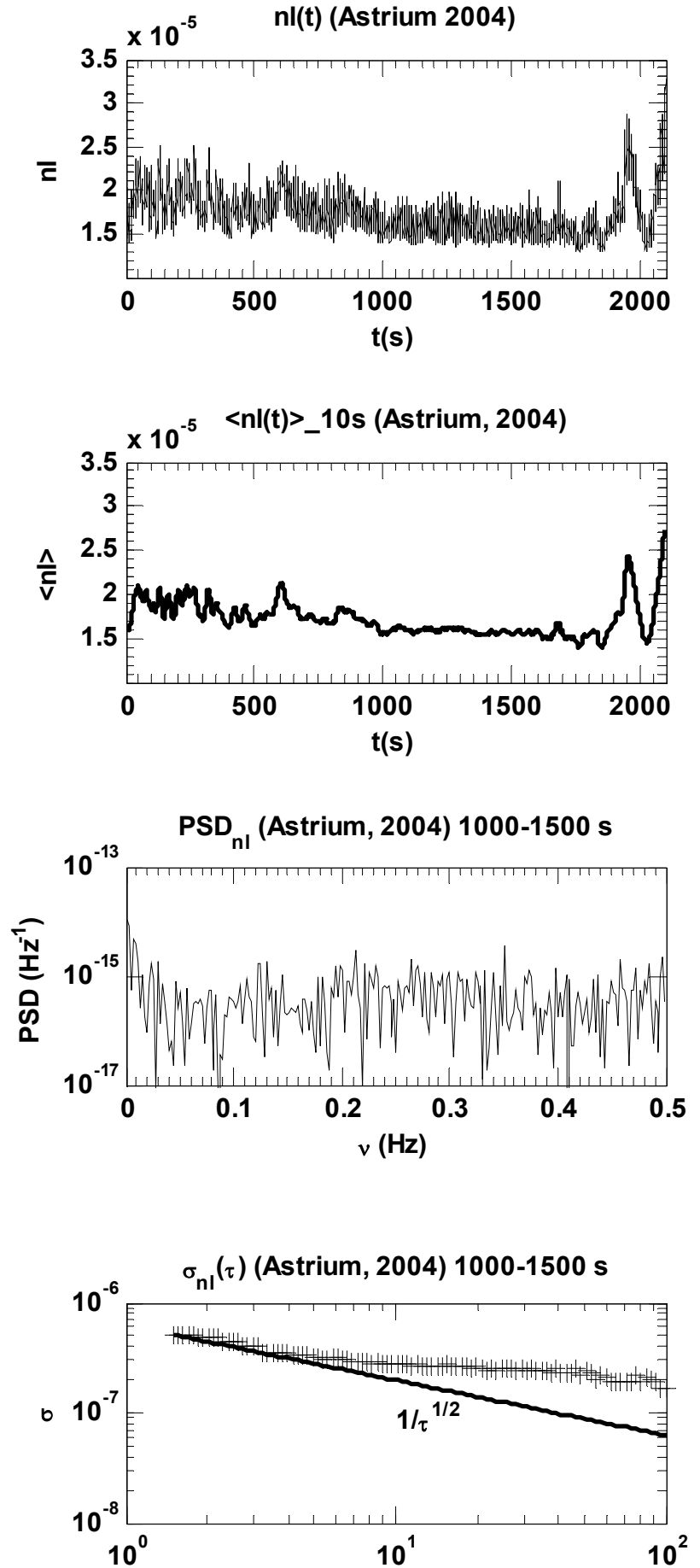

$\tau$ (s)

Figure 8 


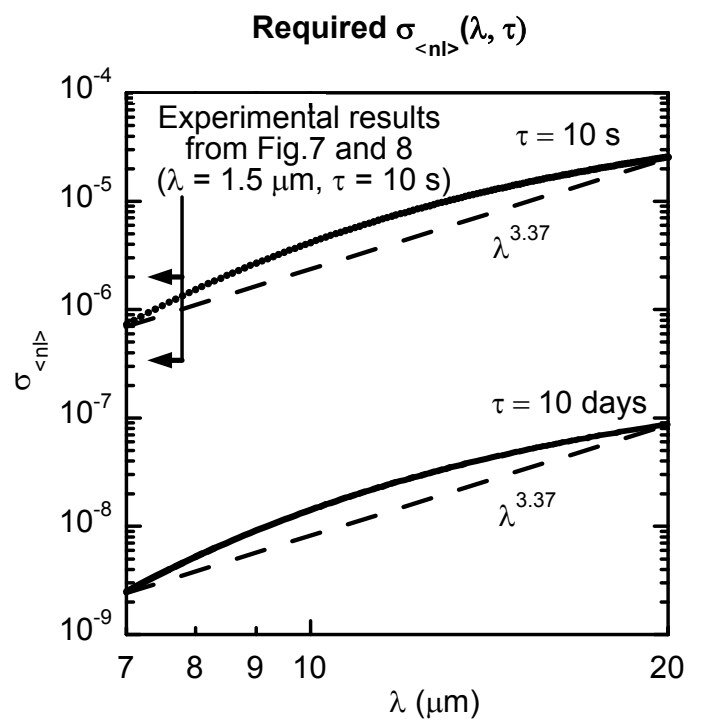

Figure 9 